\title{
Interpretation of genomic sequencing: variants should be considered uncertain until proven guilty
}

\author{
Karen E. Weck, MD
}

It is now well recognized that there are many examples of human sequence variants that had been considered pathogenic or likely pathogenic that have subsequently been reclassified. A large proportion of these have been reclassified as benign or likely benign. ${ }^{1,2}$ There are multiple reasons for reclassification and downgrading of variants, but the most powerful tool has been the cataloging of population variants with significant minor allele frequency in certain subsets of the human population, owing to efforts such as the Exome Aggregation Consortium (ExAC) and now the Genome Aggregation Database (gnomAD).

We have an ethical imperative in medical genetics not to overclassify the pathogenicity of variants because this has significant potential to cause downstream harm to patients. Otherwise, we run the risk of genomic sequencing being perceived as a flawed technique with limited clinical utility.

In interpreting the results of genomic sequencing analysis, sequence variants should therefore be considered "uncertain until proven guilty." While I applaud the recent efforts in correction of variant classification, it would be better if false calls of pathogenicity were not made in the first place. It is better to be uncertain than to be wrong.

Similar caution should be applied in reporting novel genes associated with genetic disease in individual patients. Bigger is not always better when it comes to selection of which genes to interrogate. A tiered approach to clinical genomic analysis, targeting initial analysis on genes well known to be associated with a particular phenotype, will improve the positive predictive value of genomic sequencing and reduce the likelihood of false ascertainment. There are several types of uncertainty inherent to diagnostic genomic sequencing not limited to variants, including gene evidence and phenotypic spectrum. Uncertainty at any level should be appropriately conveyed when reporting results.

\section{POTENTIAL HARMS ASSOCIATED WITH INCOR- RECT CLASSIFICATION OF VARIANTS}

The tendency toward overclassification of variant pathogenicity has adversely affected the specificity of genomic sequencing, while inflating claims of sensitivity. Multiple clinical laboratories and research studies have advertised their high sensitivity or diagnostic "hit rate" and in some cases have advertised low rates of reporting variants of uncertain significance (VUS). This is dangerous. It is more likely that the laboratories with higher rates of VUS are in fact doing a better job with variant classification, as borne out by the large number of variants previously considered pathogenic that have been downgraded in pathogenicity classification.

Avoid ascertainment bias. Even the most conservative among us have made the error of assuming that variant(s) in a gene that apparently fit a patient's phenotype must be the genetic explanation, which then turned out not to be the case once additional information was available. Imagine how many cases have been falsely ascribed but not yet corrected.

The potential harms of overclassifying variants as pathogenic include (i) making an incorrect diagnosis in an individual, which may prevent further testing to identify the correct diagnosis and/or result in ineffective treatment; (ii) conducting family studies that erroneously assign risk to relatives, which may lead to inappropriate screening or actions; (iii) making reproductive decisions based on incorrect information; ${ }^{3}$ and (iv) false annotation of variants in the literature and/or variant databases, which may affect interpretation of future patient results.

There is also an imperative for clinicians and laboratorians not to assume VUS to be benign. This would alleviate some of the possible harm in underclassifying variants; for example the reporting of a VUS as the recent subject of a lawsuit, wherein the plaintiff claimed that there was enough evidence that the variant should have been considered pathogenic (Williams v. Quest Diagnostics, Inc., et al.). Although the majority of variants classified in ClinVar as VUS will likely be reclassified as benign population variants, ${ }^{2}$ if the VUS category is used properly a significant proportion of VUS will in fact be true pathogenic variants. Clinicians should not assume that a reported VUS is benign and is therefore not associated with disease in an individual patient.

VUS are neither benign (innocent) nor pathogenic (guilty) until proven to be one or the other. It thus follows that for diagnostic testing, any VUS in a gene plausibly associated with a patient's phenotype should be reported. The decision not to report VUS for diagnostic testing predicates that

Department of Pathology and Laboratory Medicine, Department of Genetics, University of North Carolina, Chapel Hill, North Carolina, USA. Correspondence: Karen E. Weck (kweck@unc.edu)

Submitted 18 December 2017; accepted 18 December 2017; advance online publication 1 February 2018. doi:10.1038/gim.2017.269 
variant interpretation is static, knowable, concordant, and that there is a currently known "right answer." None of these things is true at this time for the extent of human genetic variability. The VUS category is a holding pattern until sufficient information becomes available to properly categorize each variant as either pathogenic or benign, although hypomorphic and risk variants may not easily fit either category.

\section{IMPLICATIONS FOR REPORTING OF SECONDARY FINDINGS}

The problem with classification of variants also has important implications for reporting of secondary or incidental findings. This is especially true for cardiac conditions such as arrhythmia and cardiomyopathy, wherein a large proportion of individuals who would be predicted to be affected, based on identification of an apparently pathogenic variant, have no evident signs of disease. ${ }^{4-7}$ In addition to a lack of understanding of the penetrance of many of these conditions in healthy or asymptomatic individuals, it is clear that the overclassification of variants has contributed to the problem. Both of these issues limit the clinical utility of reporting genomic findings in healthy or unaffected populations. ${ }^{8}$

The UK Genomic Medicine Multidisciplinary Team and 100,000 Genomes Project have debated whether there is sufficient evidence and clinical utility to report secondary genomic findings. ${ }^{9}$ They also argue that the decision of which secondary findings to return should be based at the variant level, not just the gene level. I agree strongly with this argument. A list of genes to report as secondary findings oversimplifies the reality that not all variants have the same effect, that penetrance may vary for different variants, and that limiting analysis to "known pathogenic" variants may not be practical and is fraught with overclassification errors.

Most agree that VUS should not be reported as secondary findings, and thus the overcalling of variants in this setting has crucial implications. This also applies to carrier screening for which discrepancies in variant classification may have reproductive implications. ${ }^{10}$ While there is some danger in considering variants "not pathogenic until proven otherwise," as this implies that they are likely benign, in the setting of reporting of secondary or incidental findings this is appropriate, given that the prior probability of finding pathogenic variant(s) is low in this context.

\section{ONGOING EFFORTS TO IMPROVE THE APPLICA- TION OF CLINICAL GENOMIC SEQUENCING}

We should recognize and be transparent with clinicians and patients that large-scale genomic sequencing for diagnosis is still a revolutionary technique with yet undemonstrated clinical utility. The National Human Genome Research Institute/National Cancer Institute/ National Institute on Minority Health and Health Disparities-funded Clinical Sequencing Evidence-generating Research (CSER2) awards acknowledge the need for demonstration of the clinical utility of genome sequencing as a crucial step in the application of this technology to clinical medicine.
As a result of the increased power for diagnostic potential, there is a tendency to use new technologies before clinical utility has been well established. However, there still remain gaps in our understanding of the clinical significance of every genetic variant. Important efforts that are ongoing include (i) large-scale genomic sequencing efforts and mapping of human genetic variability, (ii) ClinVar and other efforts for cataloging and annotation of the clinical relevance of variants, and (iii) increased efforts in functional assays. ${ }^{11}$ But each of these has specific caveats, and it is inevitable that rare or novel genetic variability with uncertain significance will continue to be identified.

To some extent, population statistics have come to the rescue. The most compelling evidence that a variant is benign is its existence in the human population at an allele frequency that is too high to be plausibly causative of disease. ${ }^{1,2}$ However, this predicates accurate knowledge of disease prevalence and penetrance. In addition, if the reference genome used for variant calling has pathogenic variants, or reference minor alleles, true pathogenic variants can be missed. ${ }^{12}$

Reinterpretation of genomic sequencing efforts have demonstrated significant changes to interpretation, primarily due to reclassification of variants since the time of the original report. As new information becomes available, more accurate variant classification will lead to improved sensitivity and specificity of genomic sequencing. However, challenges to clinical laboratories' resources for reinterpretation of sequencing results may be substantial and may not be reimbursable by payers, which may limit the practicality of implementing routine reanalysis of results. In addition, it is difficult for laboratories to ensure recontact of patients, emphasizing the need for clinician involvement in initiating reanalysis efforts. It would be better if variants were called VUS until proven otherwise, as ideally only VUS would be subject to reclassification. This would facilitate the need to avoid diagnostic errors that result in reclassification of variants from pathogenic to benign or vice versa.

\section{TOLERANCE OF UNCERTAINTY}

Nobody likes uncertainty-in life or in clinical medicine; genetic counselors and nongenetic clinicians alike are often frustrated when receiving an uncertain result. However, uncertainty is in fact an inherent aspect of medical diagnosis in general. There are multiple examples of pathologic diagnoses that convey uncertainty; for example, atypical squamous cells of undetermined significance (ASCUS) and monoclonal gammopathy of undetermined significance (MGUS). It is appropriate to report uncertainty in a diagnosis when the clinical significance is not clear, and this principle should equally apply to genomic medicine.

Several studies have indicated that patients and research subjects have overall good understanding and tolerance for uncertainty in genomic sequencing. ${ }^{13,14}$ This indicates that there is likely increased potential for harm in under- or overcalling the clinical significance of variants than there is in conveying uncertainty when reporting results. If patients and 
research subjects are able to tolerate uncertainty in these analyses, surely the medical community can as well, provided the inherent potential for uncertainty in current genomic sequencing tests is conveyed effectively prior to testing and upon delivery of results. It falls upon the genetic community to make this potential for uncertainty clear and not overhype the diagnostic potential of genomic sequencing.

Medical genetics is a field that has historically embraced new technologies and advancements in knowledge. There is huge potential and power in use of genomic sequencing for genetic diagnosis. This has already been realized to a large extent in human genetics, both in the identification of novel genes associated with genetic disorders, and in the identification of the cause of genetic disease in many individuals and families. It has been especially useful for diagnosing disorders with genetic heterogeneity and those that are clinically difficult to diagnose. It holds potential for genomic screening of healthy individuals -but the perils of overcalling the clinical significance of results loom especially large in this context and must be carefully considered before implementation. Uncertainty is an inevitable feature of being at the forefront of adapting new technologies to clinical care. We have a responsibility to fellow clinicians and to our patients to be clear when a result is clearly diagnostic and when there is some level of uncertainty.

\section{DISCLOSURE}

The author declares no conflict of interest.

\section{REFERENCES}

1. Lek M, Karczewski KJ, Minikel EV, et al. Analysis of protein-coding genetic variation in 60,706 humans. Nature 2016;536:285-291.
2. Whiffen $N$, Minikel $E$, Walsh $R$, et al. Using high-resolution variant frequencies to empower clinical genome interpretation. Genet Med 2017;19:1151-1158.

3. Yong E. Clinical genetics has a big problem that's affected people's lives. The Atlantic. 16 December 2015.

4. Andreasen C, Nielsen JB, Refsgaard L, et al. New population-based exome data are questioning the pathogenicity of previously cardiomyopathy-associated genetic variants. Eur J Hum Genet 2013;21: 918-928.

5. Murray MF. Your DNA is not your diagnosis: getting diagnoses right following secondary genomic findings. Genet Med 2015;18:1-3.

6. Van Driest SL, Wells QS, Stallings $S$, et al. Association of arrhythmiarelated genetic variants with phenotypes documented in electronic medical records. JAMA 2016;315:47-57.

7. Haggerty $\mathrm{CM}$, James $\mathrm{CA}$, Calkins $\mathrm{H}$, et al. Electronic health record phenotype in subjects with genetic variants associated with arrhythmogenic right ventricular cardiomyopathy: a study of 30,716 subjects with exome sequencing. Genet Med 2017;19:1245-1252.

8. Vassy JL, Christensen DK, Schonman EF, et al. The impact of wholegenome sequencing on the primary care and outcomes of healthy adult patients. Ann Intern Med 2017;167:159-169.

9. Ormondroyd E, Mackley MP, Blair E, et al. "Not pathogenic until proven otherwise": perspectives of UK clinical genomics professionals toward secondary findings in context of a Genomic Medicine Multidisciplinary Team and the 100,000 Genomes Project. Genet Med 2018;20:320-328.

10. Ramdaney A, Dunn DM, Weiss BR \& Rose NC. Beware the laboratory report: discrepancy in variant classification on reproductive carrier screening [letter]. Genet Med 2018;20:374-375.

11. Starita LM, Ahituv N, Dunham M, et al. Variant interpretation: functional assays to the rescue. Commentary. Am J Hum Genet 2017;101: 315-325.

12. Barbitoff YA, Bezdvornykh IV, Polev DE, et al. Catching hidden variation: systematic correction of reference minor allele annotation in clinical variant calling. Genet Med 2018;20:360-364.

13. Biesecker $B$, Klein $W$, Lewis $K L$, et al. How do research participants perceive "uncertainty" in genomic sequencing? Genet Med 2014;16: 977-980.

14. Skinner D, Roche MI, Weck KE, et al. "Possibly positive or certainly uncertain?": participants' responses to uncertain diagnostic results from exome sequencing. Genet Med 2018;20:313-319. 